\title{
Graphical State-Space Programmability as a Natural Interface for Robotic Control
}

\author{
Junaed Sattar, Anqi Xu, Gregory Dudek, and Gabriel Charette
}

\begin{abstract}
We present an interface for controlling mobile robots that combines aspects of graphical trajectory specification and state-based programming. This work is motivated by common tasks executed by our underwater vehicles, although we illustrate a mode of interaction that is applicable to mobile robotics in general. The key aspect of our approach is to provide an intuitive linkage between the graphical visualization of regions of interest in the environment, and activities relevant to these regions. In addition to introducing this novel programming paradigm, we also describe the associated system architecture developed on-board our amphibious robot. We then present a user interaction study that illustrates the benefits in usability of our graphical interface, compared to conventionally established programming techniques.
\end{abstract}

\section{INTRODUCTION}

This paper describes an approach and a corresponding software architecture for the programming and control of underwater and terrestrial robots. Our presented technique encompasses what we believe to be a novel variation of conventional mechanisms for specifying robot tasks. We examine this using a concrete implementation and an illustrative user study. In addition, we have tested this approach in the field. Our primary focus is to provide a graphical tool that allows robot programmers to generate plans of moderate complexity. These plans combine target trajectories with a set of activities and procedures to be followed, since tasks for mobile robots are often location-specific and thus involve both procedural as well as geometric (or other positional) information. For example, one might seek to have a robot circumnavigate a set of objects in the environment, collect data at distinct locations, and remain alert for further instructions while the plan is being executed. One of the canonical tasks for our underwater robotic vehicle, shown in Fig. 1, is to take pictures and video clips while surveying underwater coral reef structures.

Conventional task specification methods permit plans with actions conditioned upon position or other state variables, but they are often specified in a non-intuitive structure that makes visualization of the relationship between state values and corresponding actions difficult to appreciate. This paper introduces an alternative methodology and an associated visual mechanism for specifying these types of plans in a practical and natural manner. Our objective is to provide a general approach to facilitate programming activities.

A common phenomenon in robot programming, especially as related to sensor-based action planning, is the coupling

The authors are with the School of Computer Science, McGill University, 3480 University Street, Montréal, QC, Canada H3A 2A7 \{junaed, anqixu, dudek, gchare\}@cim.mcgill.ca

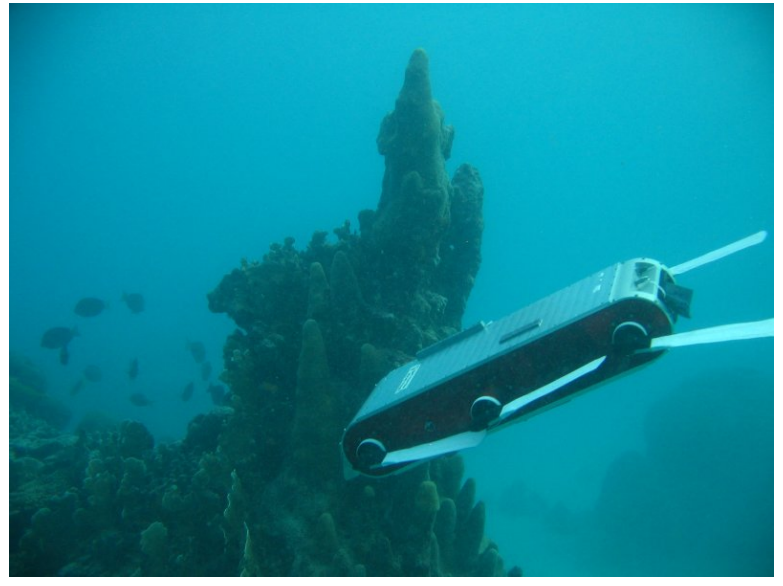

Fig. 1. Our amphibious Aqua robot exploring a populated reef environment.

of sensor processing, pose estimation and control activities. These activities are intrinsically cross-cutting, which has been demonstrated to be an impediment to understandable and maintainable code [5]. To address this concern, we propose to specify control activities using a separate graphical interface that interacts with encapsulated sensing and pose estimation modules.

Task-based robot programming often involves taking specific actions in certain locations or moving to places where events are expected to occur. Existing programming environments reflect these task specifications through three established interfaces: (1) a programmatic interface for writing procedural or functional code in a standard computer language, (2) a Graphical User Interface (GUI) for specifying trajectories or for direct teleoperation, and (3) a graphical representation of the program flow (such as an execution flowchart editor). Each of these approaches has its particular domain of application, and although they are sometimes used in combination, unfortunately their implementations often consist of graphical front-ends for fundamentally textual programming activities. Specifying position-dependent tasks using existing programming environments is accomplished by using mechanisms which amount to case-based code execution, or, in some cases, proof-theoretic conditions.

In contrast to the aforementioned approaches, this paper proposes an alternative scheme which explicitly ties code execution to regions in space using a graphical interface. By allowing users to visually indicate regions in the environment (or more generally, in the robot's state space) and by associating these coordinates with blocks of code, position- 
dependent execution becomes very easy to specify, inspect and debug. For example, to take a picture at a particular location, one simply has to attach a picture-taking script to a target region of interest within a spatial map of the robot's surroundings. Likewise, when an amphibious vehicle transitions from water onto land, the change in gait behavior can be triggered based on values of location or depth. More generally, by explicitly visualizing the connection between spatial layout and position-dependent activities, our programming paradigm assists with the separation of concerns in the context of software development ${ }^{1}$.

A secondary objective of our approach is to provide a simple scripting interface as a software wrapper to low-level control functions. This requirement helps in minimizing the amount of code needed to specify high-level commands. Our implementation provides a network-based access protocol to this scripting interface, which allows for platformindependent remote robot control.

We extended the software architecture of our amphibious robot to include the proposed state-space control scheme. This addition is particularly useful because specifying trajectories and tasks for a robot with 6 degrees of freedom is tedious and error-prone using existing interfaces such as locomotive-based control and teleoperation methods. Our graphical interface has been successfully tested in realworld marine environments on our amphibious vehicle. In particular, we used our interface to both collect sensor data and trigger condition- and state-dependent task execution.

Finally, we have conducted a simplified human interaction study to compare our proposed graphical approach to conventional textual program development. Our results illustrate the quantitative advantages of using this programming paradigm for specifying plans composed of location-dependent tasks.

\section{RELATED WORK}

Many mobile robots (and in particular exotic variants such as underwater and aerial vehicles) are programmed directly using hardware libraries and low-level Application Programming Interfaces (API). The software foundation for our own amphibious vehicle, as well as for many other stateof-the-art robotic systems, is built on top of the RoboDevel and RHexLib libraries. Unfortunately, programmers working with these libraries directly must first write a slew of basic functionalities (such as object detection and avoidance) before they can develop more sophisticated behaviors. Our proposed methodology automatically handles these mechanisms using different modules in the background to allow users to swiftly develop high-level and complex behaviors.

The Player/Stage/Gazebo robot development package [4] also provides commonly-used basic behavioral functions. The Player server establishes a standardized interface to a wide range of robot sensors and actuators over a network socket. This server is often accompanied by the Stage and

\footnotetext{
1"Separation of concerns is a well-established principle in software engineering. Nevertheless, the failure to separate concerns effectively has been identified as a continuing cause of the ongoing software crisis."[13]
}

Gazebo applications, which are visual environments for 2-D and 3-D robot simulators, respectively.

As an established successor to the Player library, the Robot Operating System (ROS) software suite [3] provides a framework for message passing, interaction scheduling and code interconnection. This development environment is targeted at building mechanisms and abstraction tools to facilitate the task of robot programming, but with a much larger scope and more amorphous goal than the approach presented by this paper. The core visualization and graphical control mechanisms provided by Player/Stage/Gazebo and by ROS are a superset of what we seek to offer, although they do not have a high-level scripting API and lack the ability to graphically bind code execution to regions in state-space.

The Microsoft Robotics Developer Studio (RDS) [6] is an Integrated Development Environment (IDE) for robotics applications. This extensive software package includes a hardware abstraction layer, a module-based communication and interaction protocol, a visual programming interface for enthusiasts, and a visual simulation environment. One of the main features of RDS is its extensive compatibility with different hardware platforms, rivaled only by a handful of other software suites such as Player and ROS. Perhaps as a direct result of its ambitious range of functions, the desire to provide fine-grained control, and the generalized application domains it targets, this rich yet complicated API can have a very high learning overhead. In contrast, our proposed scheme favors a simpler API that allows users to swiftly write code fragments at a high abstraction level.

The Subsumption architecture [1] decomposes intelligent behavior into multiple modules, each with different goals. By organizing these modules into a prioritized and layered framework, robotic systems can continuously adapt their behaviors by choosing the module that is most appropriate for its immediate neighboring environment. This approach has commonalities with our architecture (such as direct interaction with system state), although it does not require actions to relate to explicit state values.

The Saphira control system [7] is a reactive robot architecture composed of integrated routines for sensor interpretation, map building and navigation. These routines all operate on a shared representation of the robot's environment based on occupancy grids. The emphasis on a geometric world model is shared by our proposed approach.

Several robot development environments include graphical interfaces for specifying execution and data flow. These interfaces are commonly designed as learning tools for robot enthusiasts, although they also accommodate nicely to prototyping and debugging purposes. For example, the Visual Programming Language (VPL) [10] is a component of the Microsoft RDS environment that visually represent variables, robot commands and other programming constructs as interconnected blocks. By attaching blocks to each other, programmers can quickly build and deploy applications on robots while minimizing the amount of typing required. Similarly, the Lego Mindstorms NXT-G software embodies these constructs as different graphical icons, and thus provides a 
simplified interface for building basic control applications. Our approach also aims to reduce programming overhead in rapid prototyping situations, although we favor a more expressive hybrid graphical/textual representation.

The Human-Robotics Interaction (HRI) literature features many different non-conventional interaction mechanisms. The RoboChat framework [2] maps robot actions and programming constructs onto various fiducial markers. By showing these visual symbols in a particular sequence, one can program robots to accomplish complex tasks without writing a single line of code. Despite the common desire to facilitate programming, RoboChat is designed for direct robot interaction, whereas our proposed method is suited for teleoperation. Nevertheless, the simplicity of encapsulating both low- and high-level robot behaviors alike using fiducial markers inspired us to adopt a simple scripting API.

Gesture-based interfaces have been developed using both implicit and explicit communication mechanisms. Several authors have considered specialized gestural behaviors [8] (such as strokes on a touch screen) to control basic robot navigation. Skubic et al. have examined the combination of several types of human interface components to express spatial relationships and navigation tasks [12]. These natural interaction methods can be easily integrated into the presented path planning methodology to improve its usability.

\section{State-Space Programming Paradigm}

A fundamental aspect of our approach is the ability to program robot behavior using a high-level scripting language that provides access to a wide range of functions. We designed this language to provide a high degree of transparency, so that users can have access to functions implemented by the lower-level APIs. Using this substrate, we propose to combine the existing three types of robot programming methods. In particular, we allow the user to define a path in 2-Dimensional (or higher dimensional) space as a sequence of waypoints, akin to graphical control or teleoperation. The main novelty of our system is the ability to attach executable code fragments (i.e. textual programs) to regions of space or segments of the trajectory. The execution of each code fragment occurs only when the robot enters the associated waypoint or region, although execution can continue (as separate threads) for an indeterminate interval. This combination of spatial layout, preferred trajectory and code fragments is referred as a "State-Space Code Template."

By using a high-level language to specify code fragments, users can quickly attach action or behavioral routines while minimizing the amount of coding required, and without obscuring the graphical interaction. The embedded code can access primitives to load (or compute) a new trajectory, and thus conditionally-dependent path planning can be readily specified and visualized using our approach. Furthermore, by allowing code fragments to load new templates during execution, our programming mechanism allows code to be developed in layers (e.g. as contingency plans).

One or multiple inter-related State-Space Code Templates are built off-line by a robot programmer and then down- loaded onto the vehicle for execution. Unlike conventional compilation-based programming schemes, our approach allows the library of templates to be expanded during execution, thus enabling a versatile and dynamic interaction.

\section{IMPLEMENTATION}

We have implemented graphical state-space control on our family of marine vehicles, which we collectively named as Aqua. The Aqua class of vehicles is based on a hexapod body design, equipped with 3 cameras and inertial sensors, and uses six flippers to generate thrust underwater, as shown in Fig. 2. This vehicle has an aluminum body and is ballasted to be neutrally buoyant in the water. When equipped with suitable hybrid flippers, Aqua obtains limited amphibious capabilities of swimming and walking. The vehicle weighs less than $20 \mathrm{~kg}$ and operates using two general-purpose computers. Aqua operates either via remote access over a highbandwidth fiber-optic tether, using a gestural interface for specifying commands, or in a completely autonomous visual servoing setting. In all of these modes, it is commonplace to conduct trials involving an ordered sequence of experiments. Some experiments need to be repeated based on a function of external observations, internal state parameters, and the current location of the vehicle.

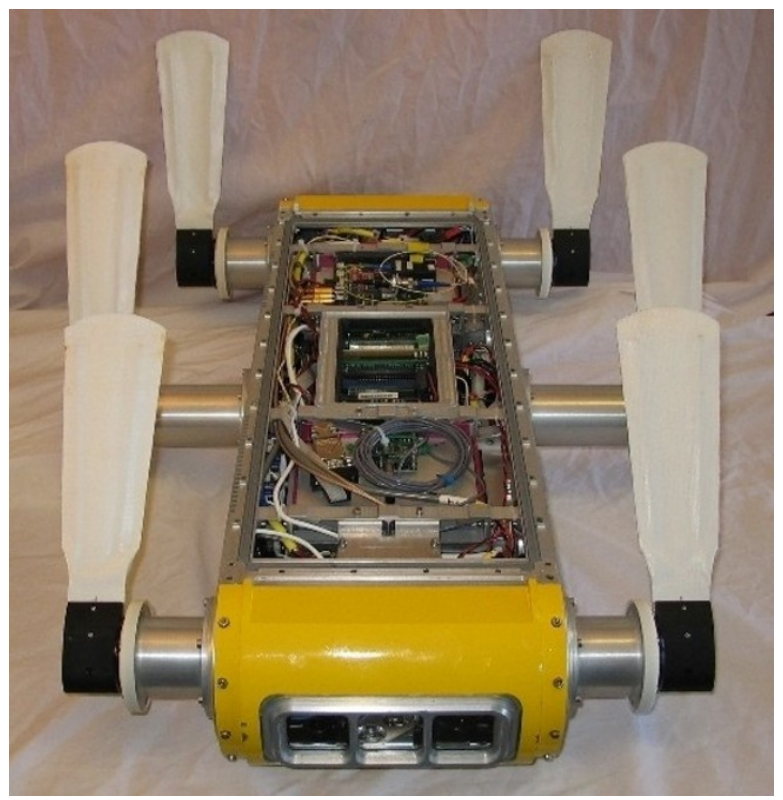

Fig. 2. The Aqua robot has 6 legs, 3 cameras ( 2 in front $\& 1$ in back), and 2 on-board processors (stacked together in the center of the chassis).

A typical task in the marine biology context is to visit a sequence of waypoints and collect data at interesting locations (e.g. by record pictures or videos). In some situations, the observations made might alter the subsequent plan of activities and trajectories. The Aqua family of robots have been deployed in regions as far south as the equator (in the Caribbean Sea) and as far north as the high Arctic ( $79^{\circ} 26^{\prime \prime}$ North latitude) to execute this type of data collection tasks. For this work we deployed the vehicle in a lake in Canada. 


\section{A. On-Board System Architecture}

In previous control schemes, Aqua is programmed and controlled using a complex API that communicates between two Operating Systems (OS) running on two distinct processors on-board the robot. The Control Stack processor interacts with hardware drivers to regulate gait control, which affects the synchronous motion model of the robot's legs. This low-level C++ controller is based on the RoboDevel [11] software suite. To meet strict real-time requirements, the control software operates on the QNX real-time OS.

The second processor inside Aqua focuses on analyzing sensory data (primarily originating from the on-board cameras), and hence is appropriately called the Vision Stack. The sensor-processing code is written in C++ and executes on top of a custom Linux OS. Since this software suite implements vision-guided motion and behavior control of the robot, it inherently provides access (via a network connection using the User Datagram Protocol, or UDP) to the low-level hardware controllers running on-board the Control Stack.

Although this control framework is highly optimized, expanding its functionality requires the use of a very complex API. To address this drawback, we developed an abstract API wrapper over the existing architecture. This RoboControl Server module is written using the Python scripting language, and provides a client-server infrastructure. This solution allows programmers to write arbitrarily-complex scripts that control the robot and receive feedback from it, without needing to modify the existing code base. Fig. 3 depicts both the hierarchy on-board the robot, as well as off-board abstraction layers implementing our proposed state-space programming paradigm.

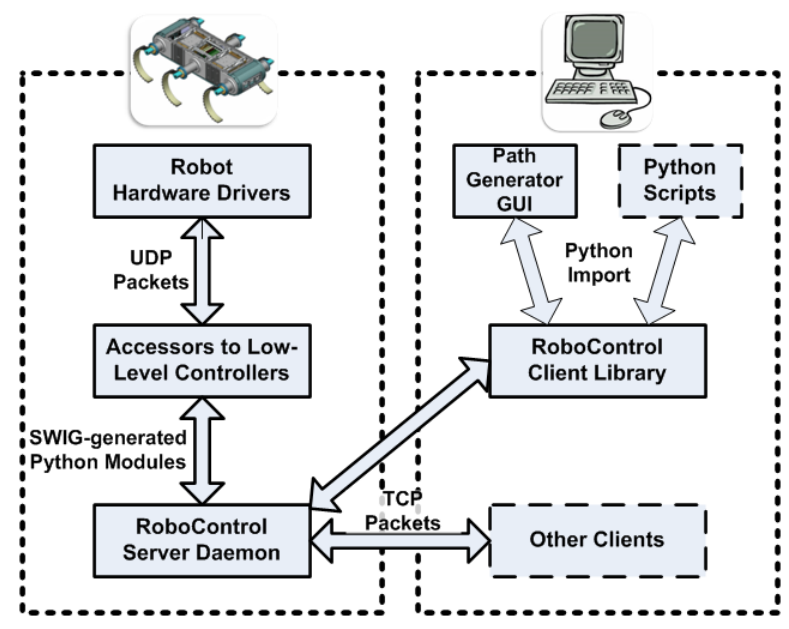

Fig. 3. Block diagram of current system architecture for the Aqua robot.

The existing API within the Vision Stack is encapsulated using an open-source C++-to-Python code generator called SWIG, which stands for Simplified Wrapper Interface Generator. We chose Python because of its abundant collection of standard libraries, its terse programming syntax, and its extended cross-platform compatibilities. To achieve both hardware and software platform independence, the RoboControl
Server provides a network interface (via the Transmission Control Protocol, or TCP) to the wrapper modules. This is accomplished by translating ASCII text received on the listening port into appropriate function calls.

\section{B. Off-Board Components}

Any application connected to the robot's network can control Aqua by sending and receiving TCP string packets to and from the RoboControl Server. In particular, we developed a Python module called RoboControl Client which is used by our state-space control GUI. This counterpart to the RoboControl Server converts Python function calls into string packets. The RoboControl Client provides an easy-touse and platform-independent interface, which allows users to control the robot using any workstation or embedded device capable of running a Python prompt.

We implemented the state-space programming paradigm as a Python-based GUI using the RoboControl Client library. This interface allows users to compose 3-Dimensional trajectories for the robot by specifying a sequence of waypoints, as shown in Fig. 4. The novelty of this GUI lies in its ability to attach Python scripts to waypoints, which are executed when the robot passes through the corresponding locations. In addition, the user can also define a global script to be executed at every waypoint.

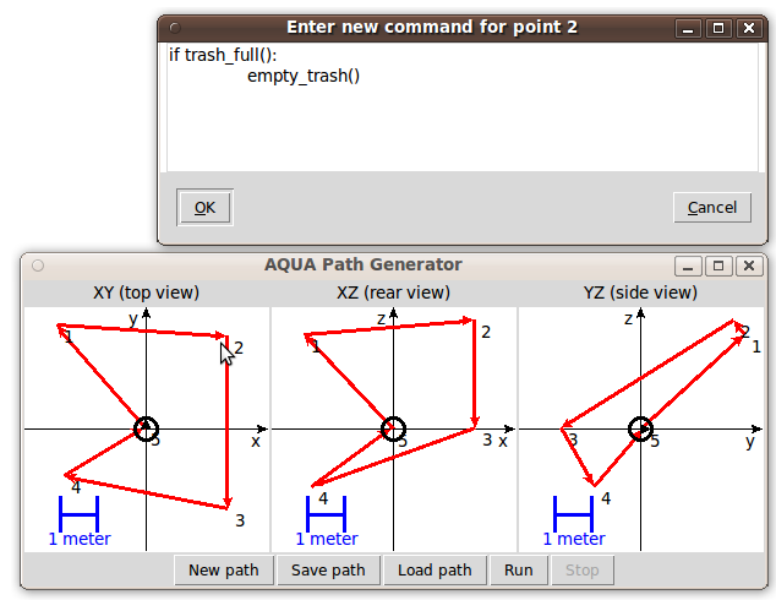

Fig. 4. A 3-D path generated using the state-space control GUI, with an attached code block for waypoint 2 .

When a path is executed using the real robot, a local planning algorithm continuously regulates the vehicle's motion to ensure that it follows the specified path. This planner can be tuned using the GUI, by adjusting parameters such as the robot's speed and directional radii of curvature to match the robot's true behaviors. The local path planner is complemented by software servos on-board the Control Stack, which compensate for unexpected forces applied onto the robot such as underwater currents.

In addition to controlling real-life robots, the GUI also includes a stand-alone simulator. In this mode, the robot's position is estimated using a predefined motion model and is updated in real-time. Any other function calls or status 
requests using the RoboControl API is automatically deferred to simulator components, which are either specified by the user or as default stubs. This simulation mode provides a powerful tool for debugging complex paths before they are executed on the real robot.

\section{EMPIRICAL VALIDATION}

We conducted a preliminary user performance study within a controlled and simulated environment to evaluate the usability of our proposed state-space programming paradigm. In particular, we compared the elapsed time required to specify a path using our GUI against the time needed to write a conventional script. Because this setup emphasizes primarily on task allocation, a simple motion model is used to facilitate programming.

We also conducted field tests with our amphibious robot using the graphical state-space interface. In particular, we used our GUI to program the robot to accomplish various tasks while moving along specified underwater trajectories. We validated these trials using qualitative and subjective performance measures.

\section{A. Study Setup}

In the controlled study, participants were asked to schedule a path for a maintenance robot through an amusement park. The layout of this fictitious environment is represented using a grid-based map, where each of the eight waypoints are depicted by icons at specific coordinates, as illustrated in Fig. 5. We used a selection of five different tasks to diversify the process and to prevent users from memorizing paths prior to each recorded attempt. To perform a task, the user must invoke a corresponding function call and attach it to the appropriate location; for example, the empty_trash() function must be called whenever the robot moves over a garbage can icon. The final code template must visit every waypoint (in any desired order) and return the robot's starting location.

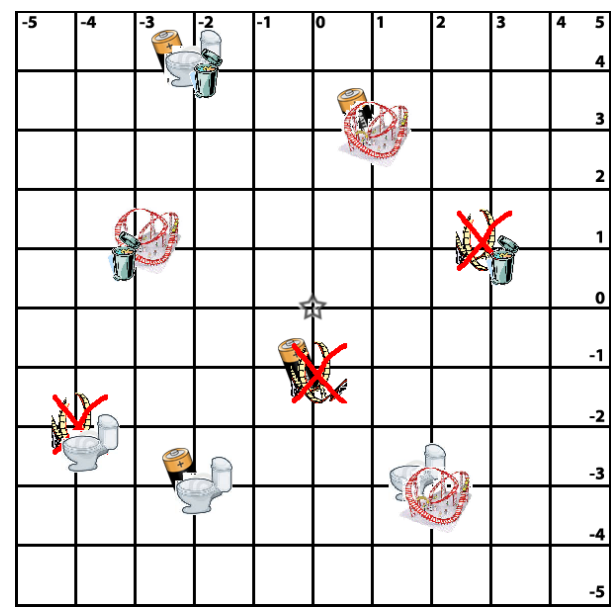

Fig. 5. Map of the environment, provided to participants of the user study.

Using our state-space programming GUI, participants assigned movement and action tasks respectively by clicking on the top-down view and by writing function calls in the appropriate pop-up dialogs. In contrast, the conventional coding approach required users to make a single sequence of function calls in plain text. Each function either performed an action, moved the robot forward by a certain number of cells, or turned the robot in-place for $90^{\circ}$ in either direction. This simplified movement model was chosen to reduce users' cognitive load during the experiment.

After a brief tutorial on how to use our graphical interface, each subject was asked to program paths twice using the GUI and twice by writing conventional scripts, in an alternating sequence. We used the elapsed time for each attempt as the evaluation metric for our study.

\section{B. Study Results}

Five participants were recruited for this study, including one female graduate student, three male graduate students and a male adjunct professor at McGill University's School of Computer Science. All subjects have varying degrees of prior programming knowledge, and although nobody had used our GUI beforehand, some had experience using similar graphical robot programming interfaces.

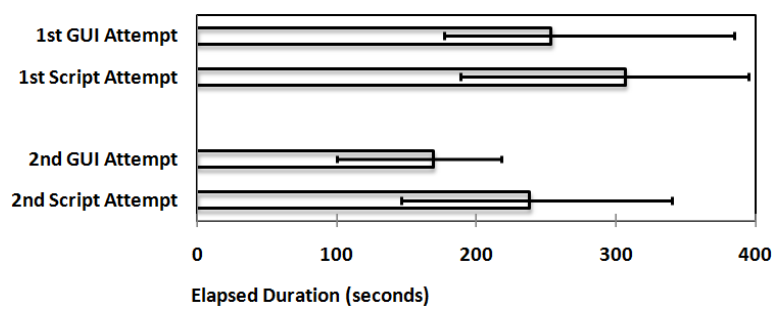

Fig. 6. Min. / mean / max. elapsed durations for programming a path using our GUI and by writing a script. Smaller values are preferred.

The results shown in Fig. 6 indicate that using the GUI lead to faster completion times on average compared to writing plain code. This performance gap is especially remarkable for the first GUI session, because the participants needed time to learn the interface. The reduced performance for the scripting method can be attributed both to users having to plan movements in the robot's local coordinate frame, which required users to keep track of the robot's current location and orientation.

We also observed that the speed increase between the GUI attempts is more prominent than for the scripting method. Since all participants had prior programming experience, the small speed increase in the second scripting session was most likely the result of familiarity with the path, due to repetition. In contrast, the participants achieved up to a two-fold speed increase in their respective GUI attempts, suggesting that our graphical interface is indeed a more natural and intuitive way for programming these types of tasks.

Anecdotally, all the participants made very few errors (and only of the typographical nature) while using the GUI. On the other hand, multiple users incorrectly moved the robot through the environment after forgetting its previous orientation. Intuitively, it is simpler to provide locations to a path planning algorithm (as in our graphical state-space 
programming paradigm) rather than manually making lowlevel function calls to move the robot.

Interestingly, some participants used copy-paste, findreplace and other editing features to reduce the amount of typing required. We are presently investigating ways of incorporating these and other common programming practices to make our graphical interface more efficient and user-friendly.

Although this human interaction study clearly illustrates the advantages of using our proposed programming paradigm, we require more elaborate and controlled studies to better assess the quantitative benefits of this new approach.

\section{Robot Field Trial}

To validate the usage of our implementation in the field, we deployed the Aqua robot in a large open-water lake. Although the results of this trial can only be reported in a qualitative form, it nevertheless allowed us to assess and verify the utility of our approach within a field deployment context. We validated both the on-board robot control using our Python API, and the use of location-specific code execution (including state-conditional execution branch and on-the-fly path loading). The latter session demonstrated the ability to re-program the robot's path in the field.

\section{CONCLUSION}

This paper described a general approach and a corresponding software tool for the graphical state-space programming of underwater and other mobile robotic vehicles. The proposed control methodology is based on the ability to associate high-level executable code to locations in physical space or in state-space. This method makes the specification of condition-dependent tasks more intuitive and more natural compared to existing programming interfaces. Moreover, by explicitly visualizing connections between states and actions, our environment enhances the design and debugging processes not only in terms of speed, but also in terms of reliability and comprehensibility. These benefits are consistent with observations made regarding the utility of graphical visualization tools for generic software engineering tasks [9]. Additionally, the visualization of both trajectories and position-dependent tasks assists with the standard software engineering objective of separation of concerns [13].

We incorporated graphical state-space control into the software architecture of our amphibious vehicle. This interface provided not only a visualization of position-dependent activities, but also introduced a transparent wrapper for low-level robot functions. Based on informal experience collected during field trials as well as a controlled user study, we observed that this interface substantially surpasses the performance of existing programming and control methods in terms of robustness, simplicity, and speed. Moreover, this approach is anecdotally observed to be a natural and pleasing development environment for creating multi-step plans (with built-in contingency) to accomplish complex tasks.

The software tool and approach we have developed can be readily extended to include other variables beyond spatial coordinates. In the case of our underwater vehicle, useful dimensions include battery voltage and the average level of local illumination. Actions conditioned on these parameters can either be programmed as waypoints (when visualizing these states) or as a plain conditional statement in the globally-executed script. Performing general state-based programming using a mixture of graphical and textual styles remains a fertile topic for further examination.

We are currently investigating ways of improving the usability of our GUI, for example by facilitating common programming practices such as macros, by overlaying the 2-D planer maps with pictures of the robot's environment, and by allowing users to define non-linear paths from one waypoint to another. Additionally, we are developing a more powerful local planner with collision-avoidance and other built-in reactive behaviors, so that users can focus their attention on high-level interactions only. Finally, we plan to deploy our programming paradigm using other natural user interfaces, such as an augmented reality setup.

\section{ACKNOWLEDGMENTS}

The authors kindly acknowledge the contribution of the participants in our human interaction study. We also gratefully appreciate the financial support of the National Science and Engineering Research Council (NSERC) of Canada.

\section{REFERENCES}

[1] Rodney A. Brooks. Intelligence without representation. Artificial Intelligence, 47:139-159, 1991.

[2] Gregory Dudek, Junaed Sattar, and Anqi Xu. A visual language for robot control and programming: A human-interface study. In IEEE International Conference on Robotics and Automation (ICRA07), pages 2507-2513, Rome, Lazio, Italy, April 2007.

[3] Willow Garage. Ros. http://www.ros.org. Accessed: 09/15/09.

[4] Brian P. Gerkey, Richard T. Vaughan, and Andrew Howard. The player/stage project: Tools for multi-robot and distributed sensor systems. 11th International Conference on Advanced Robotics, pages 317-323, 2003.

[5] Ivar Jacobson and Pan-Wei Ng. Aspect-Oriented Software Development with Use Cases (Addison-Wesley Object Technology Series). Addison-Wesley Professional, 2004.

[6] Kyle Johns and Trevor Taylor. Professional Microsoft Robotics Developer Studio. Wrox Programmer to Programmer. Wrox, 2008.

[7] Kurt Konolige, Karen Myers, Enrique Ruspini, and Alessandro Saffiotti. The saphira architecture: A design for autonomy. Experimental and Theoretical Artificial Intelligence, 9:215-235, 1997.

[8] D. Kortenkamp, E. Huber, and P. Bonasso. Recognizing and interpreting gestures on a mobile robot. In 13th National Conference on Artifical Intelligence, 1996.

[9] Peng Li and Eric Wohlstadter. View-based maintenance of graphical user interfaces. In $A O S D$ '08: Proceedings of the 7th international conference on Aspect-oriented software development, pages 156-167, New York, NY, USA, 2008. ACM.

[10] Microsoft Robotics. Vpl introduction. http://msdn.microsoft.com/enca/library/bb483088.aspx. Accessed: 09/15/09.

[11] Uluc Saranli and Eric Kavins. Object oriented state machines. Embedded Systems Programming, May 2002.

[12] M. Skubic, D. Perzanowski, S. Blisard, A. Schultz, W. Adams, M. Bugajska, and D. Brock. Spatial language for human-robot dialogs. IEEE Transactions on Systems, Man and Cybernetics, Part C, 34(2):154-167, May 2004.

[13] Stanley M. Sutton, Jr. and Isabelle Rouvellou. Modeling of software concerns in cosmos. In AOSD '02: Proceedings of the 1st international conference on Aspect-oriented software development, pages 127-133, New York, NY, USA, 2002. ACM. 